\title{
The Role of (Dis)similarity in (Mis)predicting Others' Preferences
}

\section{Citation}

Barasz, Kate, Tami Kim, and Leslie K. John. "The Role of (Dis)similarity in (Mis)predicting Others' Preferences." Journal of Marketing Research 53, no. 4 (August 2016): 597-607. doi:10.1509/ jmr.15.0226.

\section{Published Version}

10.1509/jmr.15.0226

\section{Permanent link}

http://nrs.harvard.edu/urn-3:HUL.InstRepos:37101489

\section{Terms of Use}

This article was downloaded from Harvard University's DASH repository, and is made available under the terms and conditions applicable to Open Access Policy Articles, as set forth at http:// nrs.harvard.edu/urn-3:HUL.InstRepos:dash.current.terms-of-use\#OAP

\section{Share Your Story}

The Harvard community has made this article openly available.

Please share how this access benefits you. Submit a story.

\section{Accessibility}


The Role of (Dis)similarity in (Mis)predicting Others’ Preferences

Kate Barasz, Tami Kim, and Leslie John

Forthcoming at the Journal of Marketing Research

Kate Barasz is a doctoral candidate in Marketing at Harvard Business School, Morgan Hall 180A, Soldiers Field Road, Boston, MA 02163 (email: kbarasz@hbs.edu). Tami Kim is a doctoral candidate in Marketing at Harvard Business School (email: tkim@hbs.edu). Leslie K. John is an Assistant Professor of Business Administration, Harvard Business School (email: ljohn@hbs.edu). Correspondence: Kate Barasz.

The authors gratefully acknowledge the invaluable comments and suggestions from John Gourville, Michael Norton, Todd Rogers, Lingling Zhang, and members of the GiNorton Lab. 
The Role of (Dis)similarity in (Mis)predicting Others’ Preferences

\begin{abstract}
Consumers readily indicate liking options that appear dissimilar_for example, enjoying both rustic lake vacations and chic city vacations, or liking both scholarly documentary films and action-packed thrillers. However, when predicting other consumers' tastes for the same items, people believe that a preference for one precludes enjoyment of the dissimilar other. Five studies show that people sensibly expect others to like similar products, but erroneously expect others to dislike dissimilar ones (Studies 1 and 2). While people readily select dissimilar items for themselves (particularly if the dissimilar item is of higher quality than a similar one), they fail to predict this choice for others (Studies 3 and 4) — even when monetary rewards are at stake (Study 3). The tendency to infer dislike from dissimilarity is driven by a belief that others have a narrow and homogeneous range of preferences (Study 5).
\end{abstract}

KEYWORDS: Social inference, preference prediction, self-other difference, perceived similarity, prediction error 
Suppose you encounter a consumer who is choosing between two products, Widget A and Widget B. After considering both options, the consumer decides on Widget A. Knowing of this choice, what would you predict about the consumer's preferences for forgone Widget B? Choices reveal information about people's preferences—-both for options that are chosen, as well as those that are forgone. When we observe others make a choice, we consistently assume they like the option they chose (Miller and Nelson 2002), but make more nuanced inferences about their preferences for unchosen options. As this paper demonstrates, people make different predictions about a forgone option depending on whether it is similar—or dissimilar — to a chosen option; simply put, people expect others to like similar options and to dislike dissimilar ones. Consequently, Widget B will be perceived as a well-liked substitute when it is relatively similar to Widget A, but believed to be disliked and actively rejected when it is relatively dissimilar to Widget A.

However, as we show, this intuition is often incorrect because it fails to account for the range and diversity of others' preferences: people predict others dislike dissimilar options despite recognizing that they, themselves, simultaneously like dissimilar things. For example, people readily indicate enjoying dissimilar vacation destinations (e.g., lake and city) and dissimilar movies (e.g., documentaries and thrillers), but predict that—for others—a preference for one precludes enjoyment of the other.

Five experiments demonstrate that people sensibly expect others to like similar products, but erroneously expect others to dislike dissimilar ones (Studies 1 and 2). While people readily select dissimilar items for themselves (particularly if the dissimilar item is of higher quality than a similar one), they fail to predict this choice for others (Studies 3 and 4)—even when monetary 
rewards are at stake (Study 3). We show that the tendency to infer dislike from dissimilarity is driven by a belief that others have a narrow and homogeneous range of preferences (Study 5).

\section{THEORETICAL BACKGROUND}

\section{Predicting Others’ Preferences}

The ability to accurately predict others' preferences is important across a number of contexts. We predict others' preferences when we buy gifts or make joint household decisions. Agents, such as realtors and money managers, are tasked with curating choice sets based on their clients' implicit and explicit desires. In times of medical crisis, physicians and next-of-kin are called on to make life-or-death decisions based on what they infer the patient's preferences to be. And of course, predicting customers' tastes is an essential marketing task: whether it is selecting the right merchandise to stock or promotions to run, businesses face the perennial task of making accurate predictions about their clientele.

Very often, such predictions must be made with little or no explicit information about others' actual preferences, leaving people to rely on previously observed behaviors: how has the other person chosen in the past? Consequently, previous choice information becomes influential in preference prediction. Therefore, when faced with the above question- - what would you predict about the consumer's preferences for Widget B?”- -we suggest that people engage in a discernible evaluative process, such that they: 1) attend to available information about the consumer's related choices (in this case, to the consumer's selection of Widget A); 2) evaluate the relative similarity of Widgets A and B; and 3) conclude that Widget B is liked if similar, but disliked if dissimilar (Figure 1).

[INSERT FIGURE 1] 
To elucidate, consider the first node in this evaluative process: observing a target's choice of Widget A. People readily observe one another's choices—-the cars they drive, the food they eat, the clothes they wear — and unfailingly conclude that others like the options they choose: if a consumer chooses Widget A, the consumer must like Widget A. This intuition is robust and unyielding, and persists even when the chosen option is objectively undesirable (e.g., Jones and Harris 1967; Miller and Nelson 2002). In essence, observers use others’ known choices as meaningful benchmarks against which unchosen options are compared.

This observation of choice is a necessary precondition for the proposed evaluative process; it provides a focal item against which to compare unchosen options. Without knowing that Widget A was chosen, observers would have no reason to use similarity as a basis for their predictions, and may simply project their own preferences (i.e., "I like Widget B; I predict he will like Widget B”), or evaluate Widget B on its own merits (i.e., "Widget B is good; I predict he will like Widget B”). However, we suggest that a known choice triggers the process outlined in Figure 1, such that people evaluate Widget B by comparing it to Widget A. Consequently, even if observers would find Widget B appealing and likeable in its own right—and would, in isolation, predict that it is liked — the known choice of Widget A can significantly sway their predictions.

Central to our account, this comparison process_-outlined in the second and third nodes of Figure 1-systematically shapes the specific predictions people make about others’ preferences. Research has shown that when making comparative judgments, the starting pointor "subject" — of the comparison carries special weight (Houston, Sherman and Baker 1989); people evaluate new options relative to the subject (or, in our model, to the known choice of Widget A) and pay particular attention to which attributes the new option lacks or shares. 
Therefore, the chosen option's features become a kind of de facto checklist; people note which attributes Widget B has in common with Widget A, and base their evaluations of Widget B on the degree of overlapping features (Houston and Sherman 1995; Houston, Sherman and Baker 1989).

These overlapping or common features also affect perceived (dis)similarity; items appear more similar as a function of their shared features, and less similar as a function of their distinctive or unique features (Tversky 1977). Therefore, with respect to observers’ predictions, an unchosen option that shares many features - and thus possesses many of the attributes on the desired "checklist" — will be perceived as similar and presumed to be liked, while an unchosen option that shares few features will be perceived as dissimilar and presumed to be disliked.

The surprising aspect of these hypotheses is not that people expect others to like similar items (Lefkoff-Hagius and Mason 1993); indeed, this appears to be a sensible and correct inference (Linden, Smith, and York 2003). The more interesting question is: why do people predict that others must dislike dissimilar items? Even if a dissimilar option lacks attributes on the known choice’s “checklist”- - and therefore does not possess the qualities people assume others value-it may still have features that are objectively desirable. But, as we argue, dissimilar items are not simply seen as neutral alternatives; people instead predict that they are actively rejected and disliked. Therefore, we suggest a more fundamental misperception drives this inference of active dislike: a tendency to underestimate the range and diversity of other people’s tastes. In other words, people believe others’ preferences to be more homogeneous or one-dimensional than they, in fact, are.

Researchers have documented a related tendency among groups, such that people infer out-group members are more alike than members of in-groups (e.g., Judd, Ryan and Park 1991; 
Linville, Fischer, and Salovey 1989; Park and Hastie 1987; Park and Judd 1990; Quattrone and Jones 1980). As a result of this phenomenon, the “out-group homogeneity effect,” people perceive members of an out-group to lack interpersonal diversity and be "tightly bunched around the group central tendency” (Judd et al. 1991, p. 367). As an example, a woman might believe that “all men are alike” and, correspondingly, believe that “all men are very adventurous”—or that men are narrowly distributed or "tightly bunched” around the trait of adventurousness (Rubin and Badea 2012).

Where out-group homogeneity research has shown that people see out-groups as more interpersonally homogeneous (e.g., “all men are alike”), we suggest people also perceive other individuals are more intrapersonally homogeneous (e.g., “all of that person’s preferences are alike”). Particularly once a target’s choice is known, people (mistakenly) believe all of the other person's preferences to be narrowly clustered around that single choice. For example, if an observer learns that someone chose to vacation at a lake destination, he may infer that person only likes outdoorsy vacations. We argue that this inference of homogeneity, in turn, drives the inference of dislike: narrower ranges of preferences mean that more items fall outside the realm of "what is liked," and thus have a higher probability of being disliked.

Although research has documented the persistent belief that out-group members are overly similar, it does not purport that this belief is always mistaken or erroneous (Judd et al. 1991). In the same vein, our investigation looks at the persistent belief that others' preferences are similar and narrow; we do not suggest that the belief is always incorrect. Undoubtedly, there are situations in which dissimilar may actually be indicative of dislike-for instance, someone who chose an environmentally-friendly Prius may actually hate gas-guzzling Hummers — but we document instances in which this belief is both wrong and costly (Studies 3 and 4). 
Similarity and Stimuli Selection

Because perceived (dis)similarity is central to our investigation, it is critical to articulate what qualifies as “similar” or “dissimilar.” Tversky (1977) proposed that similarity is a function of the features that are shared by two objects, and the features that are unique to one but not the other. Since then, a vast body of subsequent research—spanning cognitive, social, and consumer psychology_-has highlighted the ways in which similarity judgments can be more complex and malleable (for a review, see Medin, Goldstone and Gentner 1993); for example, similarity may vary as a function of context and option set (Dhar and Glazer 1996; Tversky and Gati 1982), time horizon (Day and Bartels 2008), characteristics and attributes considered (Houston and Sherman 1995; Lefkoff-Hagius and Mason 1993), and mode of comparison (Dhar, Nowlis and Sherman 1999; Houston, Sherman and Baker 1989).

Taking these complexities into account, we carefully selected our experimental stimuli to represent similar and dissimilar options. We used the methodology employed by past research on similarity (e.g., Boldry, Gaertner and Quinn 2007; Dhar and Glazer 1996; Lefkoff-Hagius and Mason 1993) and pretested our stimuli to gather global assessments of similarity (e.g., "On a scale from 1 to 7 , how similar do you think these two items are?”). In addition, we directly manipulated the perceived similarity of two imaginary products (Study 2) based on Tverksy’s (1977) feature-matching definition, ensuring that our results were causally related to perceived similarity, and not merely an artifact of the specific stimuli we chose. 
Five studies document when and why people infer dislike from dissimilarity. Studies 1 and 2 show the basic effect, demonstrating that people make predictions based on (dis)similarity. Studies 3 and 4 document the error in these predictions by showing that people do indeed enjoy dissimilar options for themselves_and readily choose dissimilar items that are of higher quality_-but fail to predict that others do, too. Finally, Study 5 provides evidence of the explanation underlying the prediction error: the tendency to infer dislike from dissimilarity is driven by the belief that others have a narrow and homogeneous range of preferences.

Across the five studies, we measured our dependent variable in three different-but complementary—ways to ensure the robustness of our results. We asked participants to make predictions about whether a target dislikes/is neutral about/likes a given option (Studies 1 and 2), how a target would rate an option on a 7-point Likert scale (Studies 2, 3, and 5), and how a target would choose between two options (Studies 3 and 4). Further, we asked participants to make these predictions about options that were forgone both explicitly (e.g., consumer was deciding between Widget A and B and chose A; make a prediction about Widget B) and implicitly (e.g., consumer chose Widget A; make a prediction about Widget B). Regardless of how the question was posed, and irrespective of whether the option was explicitly or implicitly forgone, the studies provide converging evidence that people infer others must dislike dissimilar things.

\section{STUDY 1}

By our account, when predicting someone’s preferences for unchosen Widget B, observers evaluate how similar Widget B is to chosen Widget A, and predict that Widget B is liked if similar and disliked if dissimilar (Figure 1). As discussed above, one necessary precondition is that a target's previous choice is known - for instance, that observers know the 
target selected Widget $\mathrm{A}$ — such that there is a focal item against which to compare unchosen options. In other words, without knowing Widget A had been chosen, observers will have no reason to use similarity as a basis for their predictions, and may predict that both Widget A and B are liked—even if they are dissimilar.

Study 1 demonstrated this dynamic by comparing a condition in which a target's previous choice was known to a condition in which a target's previous choice was unknown. We used a real-world forum for the sharing and observing of people's choices-Facebook status updatesand showed participants a post from a hypothetical friend, Joe Smith. All participants had to predict whether Joe liked or disliked two unchosen vacation options (city and mountains); in one condition, participants had no information about Joe's vacation preferences, while in another, participants learned that Joe had previously chosen to vacation at a lake. We examined how participants' predictions of Joe's preferences changed as a function of both (dis)similarity, and whether or not the previous vacation choice was known.

Procedure

Pretest. The stimuli in Study 1 were three vacation destinations: lake, mountains, and city. In the known choice condition, Joe Smith selected the lake; therefore, we selected mountains and city to appear similar and dissimilar, respectively, to that known choice. In a pretest on Mechanical Turk ( $\mathrm{N}=89$ ), we asked participants to rate the extent to which different vacation options were similar to each other $(1=$ not at all similar to $7=$ very similar $)$. The results confirmed that lake and mountains were perceived as significantly more similar $(\mathrm{M}=$ $3.31, \mathrm{SD}=1.72)$ than lake and city $(\mathrm{M}=1.69, \mathrm{SD}=.98 ; \mathrm{t}(88)=8.73, p<.001)$. 
Design. Study 1 was a two-condition, between-subjects design. Online participants $(\mathrm{N}=$ 205, 59\% male; $\left.M_{\text {age }}=31.1, S D=10.0\right)$ were either given (known choice condition) or not given (unknown choice condition) information on another person's vacation choice. To start, all participants saw the Facebook status update of a hypothetical friend, Joe Smith. In the unknown choice condition, the status update simply read: “Just booked a vacation!” In the known choice condition, the status update read: “Just booked a vacation! Headed to a lake.”

Prediction task. All participants predicted Joe Smith’s preferences for two additional vacation destinations: mountain (similar) and city (dissimilar). Participants indicated their prediction by completing two sentences, “Joe __ city vacations” and “Joe __ mountain vacations," with one of three options: dislikes, is neutral about, or likes.

This and all subsequent experiments concluded with basic demographic questions. We set the desired number of participants at the outset of the experiment, targeting recruitment of at least 100 participants per cell; for our lab sessions, slightly fewer participants showed up than were expected. We did not analyze the data until the final number of participants was reached. No data were excluded and we report all measures and conditions. All stimuli are included in the web appendix.

Results and Discussion

Prediction task. The known choice_- “Headed to a lake”— had a significant effect on participants’ predictions (Figure 2). We conducted a repeated measures logistic regression using prediction as the dependent measure; because we were most interested in the propensity to predict “dislike,” we dummy coded predictions as $1=$ dislike and $0=$ neutral or like. There was no main effect of condition ( $\mathrm{B}=-2.26, \mathrm{SE}=4.53, p=\mathrm{NS}$ ), but there was a main effect of 
destination $(\mathrm{B}=-6.93, \mathrm{SE}=1.44, p<.001)$, and importantly, also a significant interaction $(\mathrm{B}=$ 4.52, $\mathrm{SE}=1.71, p=.008)$. Follow-up tests revealed that when people predicted Joe's preferences for the similar destination, mountain, “dislike” predictions did not differ as a function of condition (B $=-.01, \mathrm{SE}=.93, p=\mathrm{NS}$ ); in both conditions, only $2.9 \%$ of participants predicted that Joe dislikes mountains. However, when predicting Joe's preferences for the dissimilar destination, city, people were significantly more likely to predict “dislike” when they knew about Joe’s previous choice of lake $(\mathrm{B}=1.51, \mathrm{SE}=.39, p<.001)$; at baseline, only $9.8 \%$ of participants predicted that Joe dislikes cities, but 33.0\% predicted that Joe dislikes cities upon learning of his dissimilar choice of lake.

\section{[INSERT FIGURE 2]}

Study 1 provides evidence that observers infer that—relative to a known choice—similar options are liked, while dissimilar options are disliked. In addition, the results also show that observers make significantly different predictions about others' preferences when a previous choice in that category is known. As a result, an option-like the city vacation-may appear perfectly likeable in one context (unknown choice) but unlikeable in another (known choice). This supports our account that a target's known choice is a necessary precondition for this inferential process, and demonstrates that observers form their predictions about unchosen options relative to known choices. (Having established this point, our remaining studies examine observers’ predictions when previous choices are always known.)

\section{STUDY 2}

Study 2 replicated and extended Study 1 in two key ways. First, we ruled out the possibility that the effect was merely an artifact of the vacation stimuli employed in Study 1 . To 
do so, we employed imaginary products, Widget A and Widget B, and directly manipulated their perceived similarity based on Tversky's (1977) feature-matching definition of similarity. Second, we included additional dependent measures to provide converging evidence of the effect.

Procedure

Design. Study 2 was a two-condition, between-subjects design. Online participants ( $\mathrm{N}=$ 297, $67 \%$ male; $M_{\text {age }}=30.7, S D=9.7$ ) read a scenario about a consumer, Jane, who was choosing between two products. In one condition, the two products were framed to appear relatively similar (similar condition); in the other condition, they were framed to appear relatively dissimilar (dissimilar condition).

All participants encountered the same basic scenario: "Suppose that Jane is choosing between two different products, Widget A and Widget B. Both Widgets can be described by 5 key attributes: price, size, shape, function, and quality." In the similar condition, participants learned: "Widget A and Widget B share 4 of 5 attributes. In other words, they have 4 attributes in common.” In the dissimilar condition, participants learned: "Widget A and Widget B share 1 of 5 attributes. In other words, they have 1 attribute in common." In both conditions, participants were informed of Jane’s choice: “Jane chooses Widget A.”

Prediction task. Participants made three predictions about Jane’s attitudes toward Widget B, the forgone option. First, participants predicted how much Jane likes or dislikes Widget B on a 7-point Likert scale $(1=$ does not like at all to 7 = likes very much). Next, as in Study 1 , participants completed the sentence, “Jane __ Widget B," by selecting one of three options: dislikes, is neutral about, or likes. Last, participants estimated how likely Jane would be to buy Widget B if Widget A were no longer available $(1=$ not at all likely to $7=$ very likely $)$. 
Manipulation check. At the end of the survey, participants assessed the perceived (dis)similarity of Widgets A and B $(1=$ very dissimilar to $7=$ very similar $)$.

Results and Discussion

Manipulation check. As intended, participants perceived Widgets A and B to be less similar in the dissimilar condition $(\mathrm{M}=2.88, \mathrm{SD}=1.47)$ than in the similar condition $(\mathrm{M}=5.82$, $\mathrm{SD}=0.80 ; t(295)=21.44, p<.001)$.

Prediction task. While Widget B was forgone in both conditions—rejected by Jane in favor of Widget A—participants made different predictions about Jane’s preferences depending on Widget B's perceived (dis)similarity to Widget A. For the first measure, participants in the dissimilar condition predicted Jane liked Widget B significantly less $(\mathrm{M}=3.25, \mathrm{SD}=1.23)$ than participants in the similar condition $(\mathrm{M}=4.70, \mathrm{SD}=1.17 ; t(295)=10.37, p<.001)$.

For the second measure, participants predicted whether Jane dislikes, is neutral about, or likes Widget $\mathrm{B}$. The overall chi-square test revealed a significant main effect of condition on preference prediction $\left(\chi^{2}(2)=78.492, p<.001\right.$; Figure 3). Most participants in the dissimilar condition (61.5\%) predicted that Jane dislikes Widget B; in contrast, few (14.8\%) participants in the similar condition predicted that Jane dislikes Widget B $\left(\chi^{2}(1)=68.76, p<.001\right)$.

Finally, when asked to imagine that Widget A was unavailable, participants in the dissimilar condition thought Jane would be less likely to buy Widget $B(M=4.11, S D=1.57)$ than participants in the similar condition $(\mathrm{M}=5.81, \mathrm{SD}=1.04 ; t(295)=11.02, p<.001)$, suggesting that different beliefs about Jane’s preferences also translated into different beliefs about Jane’s future behavior.

[INSERT FIGURE 3] 
All participants were given the same information about Jane’s preferences: she had selected Widget A over Widget B. However, as expected, participants made significantly different judgments about forgone Widget B depending on its relationship to chosen Widget A. Therefore, Study 2 makes two key contributions. First, it rules out the possibility that Study 1's results were simply an artifact of the specific stimuli we used, instead showing that the effect emerges even in a minimalist setting using a clean and crisp manipulation of similarity. Second, by documenting the effect using three different dependent measures — the sentence completion task from Study 1, a scale measure of liking, and a prediction of future behavior—-Study 2 provides converging evidence that people use dissimilarity to predict dislike.

So far, we have shown that observers make different predictions when they know (versus do not know) what someone else previously chose (Study 1), and more importantly, that they evaluate new options based on the relative (dis)similarity to that known choice (Studies 1 and 2). In Studies 3 and 4, we tested whether these inferences are accurate.

\section{STUDY 3}

Studies 3 and 4 tested the accuracy and strength of the belief that others dislike dissimilar things. To test the accuracy of this belief, both studies collected both actual and predicted preferences: participants either indicated their own preference (self condition), or—as in Studies 1 and 2-predicted someone else’s preference (observer condition). Comparing actual and predicted preferences provided a measure of accuracy.

To test the strength of this belief, both studies asked participants to make a trade-off between similarity and quality. Specifically, participants had to choose (either for themselves or someone else) between a three-star movie in a preferred genre and a five-star movie in a 
dissimilar genre. In addition, Study 3 also used an incentive compatible design with a monetary bonus awarded for predictive accuracy. We anticipated that participants would choose the higher rated but dissimilar movie for themselves, but—-despite the quality trade-off and monetary incentive-would predict others to make the opposite choice.

Procedure

Design. Study 3 was a two-condition, between-subjects design. Lab participants $(\mathrm{N}=$ 178; $52 \%$ male; $\mathrm{M}_{\text {age }}=22.6, \mathrm{SD}=3.8$ ) completed a study at a northeastern U.S. university lab in exchange for $\$ 20$ and the possibility to earn a $\$ 2$ bonus. Upon entering the lab, participants were randomly placed in dyads; one partner in the dyad reported his/her own preferences (self), while the other predicted his/her partner's preferences (observer). Dyads were seated on opposite sides of the room and remained anonymous to one another; their only interaction was through paper exchange via an experimenter.

Establishing a known choice. To begin, participants in the self condition indicated on a piece of paper which of two movies - a five-star thriller and a five-star documentary — they would prefer to watch. An experimenter collected the papers and delivered them to the partners who had been assigned the role of observer. Thus observers saw both the question and how their partner had answered. Both participants in the dyad also entered the preferred movie choice into the computer.

Ratings task. Next, we measured participants’ actual and predicted ratings. All participants provided ratings of both genres (thrillers and documentaries) on a 7-point Likert scale $(1=$ not at all to 7 = very much). Specifically, participants in the self condition were asked: 
“Generally speaking, how much do you like each genre?” Participants in the observer condition were asked: “Generally speaking, how much do you think your partner likes each genre?”

Trade-off task. Next, both partners were presented with a trade-off choice task.

Participants in the self condition were told: "Suppose that your first-choice movie is no longer available. Which movie would you select instead?” These participants had the option of a lower quality, three-star version of their preferred genre (as established in the known choice task) or a higher quality, five-star version of the unchosen, dissimilar option. Specifically, had a participant preferred thrillers, her trade-off choice would be between a three-star thriller and a five-star documentary; had the participant preferred documentaries, her trade-off choice would be between a three-star documentary and a five-star thriller.

In the observer condition, each participant predicted his/her partner's choice from the same two options (i.e., a three-star version of the preferred genre or a five-star version of the dissimilar, unchosen genre). Observers were told: “Suppose that your partner’s first-choice movie is no longer available. Making a decision on your partner's behalf, which movie would you select instead?” In addition, they were told: “If your answer matches your partner’s selection, you will receive a $\$ 2$ bonus upon checkout.”

Preference strength task. As one final measure, participants in the self condition indicated how much they liked the two trade-off options (three-star version of preferred genre and five-star dissimilar option) after they had made their trade-off choice. These participants rated each option on a 7-point Likert scale $(1=$ not at all to $7=$ very much $)$. This measure was designed to elucidate the magnitude of the prediction error, determining whether "mistaking dissimilar for dislike” results in choosing something on someone's behalf that is slightly—or considerably—less preferred. 
Results and Discussion

Ratings task. A 2 (between-subjects, role: self versus observer) x 2 (within-subjects, option: preferred versus dissimilar option) mixed ANOVA revealed two main effects.

Unsurprisingly, regardless of role, the preferred option (as indicated in the known choice task) was rated significantly higher than the unchosen, dissimilar option $\left(\mathrm{M}_{\text {preferred }}=5.46, \mathrm{SE}=.07\right.$; $\left.\mathrm{M}_{\text {dissimilar }}=3.47, \mathrm{SE}=.10 ; \mathrm{F}(1,176)=439.08, p<.001\right)$. There was also a main effect of role $\left(\mathrm{M}_{\text {self }}=4.83, \mathrm{SE}=.10 ; \mathrm{M}_{\text {observer }}=4.10, \mathrm{SE}=.10 ; \mathrm{F}(1,176)=27.20, p<.001\right)$.

Critically, these main effects were qualified by a significant interaction $(F(1,176)=$ 23.56, $p<.001)$. Specifically, when rating the preferred option, the difference between self and observer ratings was small in magnitude and marginal in significance $\left(\mathrm{M}_{\text {self }}=5.60, \mathrm{SD}=1.00\right.$; $\left.\mathrm{M}_{\text {observer }}=5.33, \mathrm{SD}=.89 ; \mathrm{t}(176)=1.91, p=.06\right)$. However, observers’ predicted ratings for the unchosen, dissimilar option were substantially and significantly lower than their partners’ actual ratings $\left(\mathrm{M}_{\text {observer }}=2.88, \mathrm{SD}=1.01 ; \mathrm{M}_{\text {self }}=4.07, \mathrm{SD}=1.51 ; \mathrm{t}(176)=6.18, p<.001\right)$. Assessed another way, self participants reported a smaller difference in ratings between their preferred and unchosen options $(\mathrm{M}=1.53, \mathrm{SD}=1.37)$ than observer participants predicted $(\mathrm{M}=2.45, \mathrm{SD}=$ 1.16; $\mathrm{t}(176)=-4.85, p<.001)$. In addition, observer ratings for the dissimilar option were significantly below the scale midpoint $(\mathrm{t}(88)=-10.50, p<.001)$, while self ratings were not $(\mathrm{t}(88)=.42, p=.68)$.

\section{[INSERT FIGURE 4]}

Trade-off task. All participants were asked to choose-either for themselves or on behalf of their partner-between a three-star version of the preferred genre and a five-star version of the dissimilar, unchosen genre. While most (68.5\%) participants chose the higher quality, dissimilar 
option for themselves, fewer than half (39.3\%) of those in the observer condition predicted that choice $\left(\chi^{2}(1)=15.29, p<.001\right)$ —despite having been incentivized for accuracy.

Preference strength task. Participants in the self condition not only selected the higher quality dissimilar option in the trade-off choice task; they also strongly preferred the higher quality, dissimilar option $(\mathrm{M}=4.83, \mathrm{SD}=1.59)$ to the lower quality version of the preferred genre $(\mathrm{M}=3.66, \mathrm{SD}=1.28 ; \mathrm{t}(88)=5.37, p<.001)$. Additionally, self participants rated the higher quality, dissimilar option significantly above the scale midpoint $(\mathrm{t}(88)=4.93, p<.001)$, and the lower quality version of the preferred genre significantly below the scale midpoint $(\mathrm{t}(88)$ $=-2.49, p=.02$ ). This result suggests that the prediction error is considerable in magnitude: observers not only mispredicted their partners' choices, but also predicted an option that was liked significantly less.

Study 3 demonstrated that observers both misestimated others’ ratings and mispredicted others’ choices, believing both that dissimilar items were liked significantly less than they actually were, and predicting a rejection of the higher quality, dissimilar option in favor of a lower quality, similar option. Moreover, observers made this error despite being financially incented for accuracy.

\section{STUDY 4}

Study 4 tested the specificity of the error. As in Study 3, participants chose between two movies of varying quality; however, unique to Study 4, we used both a pair of dissimilar genres (i.e., thriller and documentary) and a pair of similar genres (i.e., thriller and action adventure). We expected participants to believe that others can like similar options, and thus predict others 
would be willing to switch to a higher quality, similar genre. Critically, however, we anticipated that participants would fail to predict the switch when the alternative option was dissimilar.

Procedure

Pretest. Study 4 used a similar pair of movies (i.e., thriller and action adventure) and a dissimilar pair of movies (i.e., thriller and documentary). A separate pretest confirmed that thrillers and action adventures are perceived to be more similar $(\mathrm{M}=4.45, \mathrm{SD}=1.46)$ than thrillers and documentaries $(\mathrm{M}=2.03, \mathrm{SD}=1.28 ; p<.001)$.

Design. Study 4 was a 2 (role: self versus observer) x 2 (option set: similar vs. dissimilar) between-subjects design. Online participants ( $\mathrm{N}=601 ; 64 \%$ male; $\left.\mathrm{M}_{\text {age }}=32.7, \mathrm{SD}=10.6\right)$ first encountered a known choice task followed by a prediction task. To manipulate role, we asked participants to either report their own preferences (self) or to predict someone else's preferences (observer). To manipulate option set, we asked participants to either choose between two similar movie genres or two dissimilar movie genres.

Establishing a known choice. Participants in the self condition indicated which of two movie genres they preferred (“In general, which movie genre do you prefer?”). Half of these participants (i.e., one-fourth of all participants) selected between two similar options, thriller and action adventure; the other half selected between two dissimilar options, thriller and documentary. To make the genres tangible, we included three well-known and contemporary examples of each (thriller examples: Gravity, Captain Phillips, Lone Survivor; action adventure examples: The Dark Knight, Terminator, Star Trek; documentary examples: Blackfish, Man on Wire, Inside Job.) 
Participants in the observer condition were told which of the two genres someone else preferred. Specifically, observers read: “Study participant (Participant \#62013) was asked the following question and provided the following answer.” (In reality, we randomly assigned participants to receive different information about Participant \#62013's preferences.) A screenshot of the survey question was displayed, and a radio button was filled in to indicate the previous respondent's selection. Half of the observer participants saw Participant \#62013’s selection between two similar options, thriller and action adventure (counterbalanced); the other half saw Participant \#62013’s selection between two dissimilar options, thriller and documentary (counterbalanced). To ensure that participants had processed this information, observer participants were asked: “Which movie genre does Participant \#62013 generally prefer?” They were not able to advance until they had correctly restated the choice.

Trade-off choice. Next, participants faced a choice, either making it for themselves (“Which movie would you rather see?”) or predicting it for someone else (“Which movie do you think Participant \#62013 would rather see?”). As in Study 3, participants chose between a threestar version of the preferred genre (as established in the known choice task) and a five-star version of the alternative genre.

Results and Discussion

Trade-off choice. A logistic regression revealed that the propensity to choose the higher quality movie depended on both role $(\mathrm{B}=2.09, \mathrm{SE}=.27, p<.001)$ and option set $(\mathrm{B}=2.29$, SE $=.28, p<.001)$. More importantly, the predicted interaction between role and option set emerged $(\mathrm{B}=-1.86, \mathrm{SE}=.37, \mathrm{p}<.001$, Figure 5). Follow-up tests revealed that when the option set consisted of similar movies (i.e., thriller and action adventure, left side of Figure 5), there was no 
difference in actual and predicted choice (self $=73.5 \%$ vs. observer $=68.7 \% ; \chi^{2}(1)=.86, p=$ .35), demonstrating that participants correctly predicted others' trade-off choices among similar items. However, when the option set contained dissimilar movies (i.e., thriller and documentary), a significant prediction error emerged. While most people (64.2\%) preferred the higher quality, dissimilar movie for themselves, most observers failed to predict this preference: only $18.1 \%$ correctly predicted that others would select the higher quality dissimilar movie $\left(\chi^{2}(1)=65.78, p\right.$ $<.001)$.

\section{[INSERT FIGURE 5]}

While Study 3 documented the error in the prediction, Study 4 went further by demonstrating the specificity of the error: although people inaccurately predicted others' choices between dissimilar options (i.e., thriller and documentaries), their predictions were accurate when the options were similar (i.e., thriller and action adventure). Having demonstrated that observers mistakenly predict dissimilar items must be disliked, Study 5 shows why this effect occurs.

\section{STUDY 5}

We propose that the tendency to infer dislike from dissimilarity is driven by a belief that others have a narrow and homogeneous range of preferences, and therefore must not like dissimilar options. We draw on research from out-group homogeneity to support this account: where out-group homogeneity research documents the belief that out-group members lack interpersonal variability (e.g., "all men are alike”), we suggest people also believe other individuals lack intrapersonal variability (e.g., "all of that person's tastes are alike”). In turn, this perceived lack of variability drives people to think that others' preferences are mutually 
exclusive: if someone likes documentaries or lake vacations, he necessarily must not like dissimilar "opposites," thrillers or city vacations. Narrower and less variable preferences mean that more items fall outside the realm of "what is liked," and have a higher probability of being disliked.

To test this account, Study 5 measured individuals' beliefs that others have homogeneous preferences using a distribution task akin to those used in out-group homogeneity research (Boldry, Gaertner and Quinn 2007; Judd et al. 1991; Linville et al. 1998; Park and Hastie 1987; Park and Judd 1990). As an example, Park and Hastie (1987) asked participants, "How many of these 100 [out-group] members would fall into each of eight boxes, which represent a continuum of the trait dimension intelligence," where the boxes on the far left and right were labeled "stupid” and "intelligent,” respectively; smaller ranges and tighter distributions reveal perceptions of homogeneity. We adapted this approach to directly measure participants' beliefs about the distribution of others' preferences in a particular domain: we asked participants to estimate the distribution of an average consumer's music preferences across multiple genres. Narrower distributions indicated a belief that others' preferences are homogeneous and onedimensional, while broader distributions indicated a belief that others' tastes are more diverse and varied.

Anticipating variability in the strength of this belief, we then examined the role of the measured belief in how people make predictions. We replicated the basic design of Study 1, in which participants predicted how much a target likes a mountain and city destination after learning of his/her choice of a lake vacation. We expected that people who strongly believe others have homogeneous tastes would be more likely to predict that a dissimilar option is 
disliked. Inversely, we anticipated that for people who believe others have diverse tastes, the effect would be mitigated and dissimilar options would be judged more favorably.

Procedure

Design. Study 5 was a two-condition, within-subjects design manipulating option (similar versus dissimilar). A second factor, belief in preference homogeneity, served as a measured independent variable. Online participants $\left(\mathrm{N}=196 ; 59 \%\right.$ male; $\left.\mathrm{M}_{a g e}=34.3, \mathrm{SD}=11.6\right)$ were all asked to take an observer role and make predictions about someone else's preferences.

Measuring the belief. To begin, all participants read the following: "Imagine that an average U.S. consumer was asked to indicate his/her music preferences by telling us how many songs from each music genre he/she would put in a 5-song playlist. We have disguised the specific music genres, but imagine the options included 5 very different kinds of music: Alternative Rock, Top 40 Pop, Classical, American Folk, and Hip Hop. How many songs do you think he/she would select from each genre?” Below the question, participants saw 5 textboxes with "Genre 1" through "Genre 5" labeled next to each; participants specified the number of songs from each genre, summing to five total songs. The distribution indicated participants' beliefs about others' preference homogeneity; for example, a distribution of $[0,5,0,0,0]$ indicated a strong belief about another person's preference homogeneity (i.e., tightly clustered and unvaried tastes), while a distribution of $[1,1,1,1,1]$, indicated a weak belief in preference homogeneity (i.e., dispersed and varied tastes).

Prediction task. On the following screen, participants were informed that they would next be asked to make predictions about a different person's vacation preferences. Rather than predicting for an average U.S. consumer, participants now made predictions for a "previous 
survey respondent.” (In reality, we created the answer this respondent had supposedly provided.) All participants read: "In a previous survey, we asked participants about their previous vacation choices. Participant \#91811 provided the following response: Lake.” As the dependent measures, all participants were asked to predict how Participant \#91811 had answered the following two questions: "How much would you like or dislike taking a trip to a city destination?” and “How much would you like or dislike taking a trip to a mountain destination?” Participants predicted the target's response on a 7-point Likert scale $(1=$ dislike very much to $7=$ like very much).

\section{Results and Discussion}

To measure the belief in preference homogeneity, we followed an approach used in outgroup homogeneity literature and computed the standard deviation for each participant's allocation (e.g., Linville et al. 1989); higher "belief scores” represent stronger beliefs in preference homogeneity, while lower scores represent weaker beliefs in preference homogeneity. (For example, a relatively narrow distribution of $[0,1,4,0,0]$ would be assigned a belief score of 1.73 , while a relatively diverse distribution of $[0,1,1,1,2]$ would be assigned a belief score of 0.71.) The maximum possible (and observed) value was 2.24, and the minimum possible (and observed) value was 0.00. The mean belief score was $1.06(\mathrm{SD}=0.51)$.

To analyze the data, we used the method proposed by Spiller et al. (2013) to test for simple effects in a 2 (within-subjects) by continuous design. We created a contrast score for each participant by computing the difference in predicted ratings between the similar option, mountain, and the dissimilar option, city $\left(Z_{\text {contrast }}=Y_{\text {mountain }}-Y_{\text {city }}\right)$. A regression was performed using the contrast score as the dependent variable, and belief in preference homogeneity as the independent variable. The results indicate a significant simple effect of option $(\mathrm{B}=2.20, \mathrm{SE}=$ 
$.29, p<.001$ ), such that participants on average predicted higher ratings for mountain than city. The results also indicate a significantly positive slope for the measured variable, belief in preference homogeneity $(\mathrm{B}=.76, \mathrm{SE}=.25, p=.002)$, indicating that the gap between ratings increased as beliefs in preference homogeneity strengthened. To further explore this interaction, we examined the slopes of predicted ratings for both mountain and city. While the slope of mountain ratings was positive but not significant $(\mathrm{B}=.26, \mathrm{SE}=.16, p=.10)$, the slope of city ratings was both negative and significant $(\mathrm{B}=-.49, \mathrm{SE}=.17, p=.005)$. In other words, people who tended to believe others' tastes are homogeneous were also significantly more likely to infer that dissimilar entails dislike.

\section{[INSERT FIGURE 6]}

Study 5 provides evidence for why people infer dislike from dissimilarity. As our account suggests, people default to the belief that others have relatively narrow and homogeneous preferences, and thus predict that dissimilar items are disliked. Our results confirm that the people who hold this belief most strongly are also the ones who predict the lowest ratings for dissimilar options. One possible limitation is that we elicited participants’ preference homogeneity beliefs immediately prior to their preference predictions, which could have produced unintended spillover effects; however, such a limitation is likely mitigated by the fact that participants answered on behalf of two explicitly different targets (i.e., "an average U.S. consumer” versus “Participant \#91811”) about two different product categories using two distinct measures. 
Five studies demonstrated that despite liking dissimilar options themselves, people routinely predict that others do not. Relying on others' known choices, people predict that others will like similar options and dislike dissimilar ones (Studies 1 and 2). However, comparing predicted preferences to actual preferences, the error in this heuristic becomes clear: people readily select dissimilar items for themselves (particularly if the dissimilar item is of higher quality than a similar one), but fail to predict this choice for others (Studies 3 and 4)—even when monetary rewards are at stake (Study 3). Further, we show that the prediction error is driven by the misguided belief that others’ preferences are homogeneous (Study 5).

Our basic paradigm—drawing inferences from someone’s choice—is pervasive in everyday life; we routinely acquire information about other people's decisions and make judgments accordingly. Consequently, this prediction error may also be pervasive, leaving us to extrapolate more than is warranted by the bits of information we encounter. Further, these erroneous inferences happen even for options that are only implicitly (versus explicitly) forgone; for example, participants in Studies 1 and 5 knew only that the target had chosen a lake vacation—not that the target had chosen the lake vacation over a city vacation—and yet, these participants still overwhelmingly inferred that cities were disliked because of their dissimilarity.

Particularly in contexts like recommendation-making and gift-giving, this bias may materially change what people select for someone else: if people are prone to making the wrong inferences about dissimilar options — even those options about which they have no explicit information—-they may miss opportunities to diversify and optimize their recommendations or gifts. For instance, might a realtor forgo the opportunity to show a well-priced contemporary property after learning of her client's interest in a traditional home-mistaking a preference for the latter as an indication of dislike for the former? Or consider even more consequential 
contexts: knowing that a terminally ill patient had previously chosen an aggressive and experimental curative treatment, might a physician infer that her patient therefore rejects the more passive palliative option, and fail to initiate a more comprehensive discussion of all possible treatments?

Beyond actively mispredicting others' preferences, we suggest this mistaken inference has broader interpersonal implications, particularly in situations where it is important to gauge others' intentions. For example, consider what might happen if, in the course of a high-stakes negotiation, two dissimilar but equivalently viable options are put forth; would the other party's explicit endorsement of one option signal an implicit rejection of the other- even if that forgone option were only slightly less preferred? It is easy to see how this incorrect inference could lead to hostile or stalled negotiations. As a final example, one of us had a brief moment of panic when a senior colleague pointed out how different it was to collaborate with her versus another of his frequent coauthors; she fretted momentarily, assuming that if her colleague liked working with one, he could not possibly like working with the other, and worried that she might be the dissimilar-and-therefore-disliked collaborator. But, recalling the takeaways of her own research, she realized (or at least hoped!) that she was underestimating her colleague’s preferences for a diverse array of coauthors.

As a final thought, we propose that marketers, gift-givers, and recommenders should not constrain themselves to exclusively offering or suggesting similar products or options. While complex algorithms may steer marketers’ product recommendations in Big Data-enabled settings (Linden, Smith and York 2003), there are myriad situations in which people give real-time, faceto-face, non-analyzed recommendations. Although it may be more difficult to predict which dissimilar items will have the most positive reception, there may be valuable upside to "going 
out on a limb” and suggesting something seemingly different. For example, someone who just watched a thriller may be bored by the obvious recommendation for yet another thriller —in which case the recommender risks appearing incapable of generating more nuanced and customized ideas; perhaps instead offering an acclaimed documentary could both pique consumer interest and make the recommender appear savvy and sophisticated. As our studies suggest, people are willing to sample diverse options, and in an environment where recommendations are routinely and recognizably based on item-to-item similarity, firms and individuals may risk appearing trite or unoriginal—or worse, like they have failed to recognize the variety of their clients' tastes. In this case, understanding that dissimilarity does not entail dislike may help people cater to the diversity of others’ preferences. 


\section{REFERENCES}

Boldry, Jennifer G., Lowell Gaertner, and Jeff Quinn (2007), “Measuring the Measures: A MetaAnalytic Investigation of the Measures of Outgroup Homogeneity,” Group Processes \& Intergroup Relations, 10 (2), 157-78.

Day, Samuel B. and Daniel M. Bartels (2008), "Representation over time: The effects of temporal distance on similarity,” Cognition, 106 (3), 1504-13.

Dhar, Ravi and Rashi Glazer (1996), "Similarity in Context: Cognitive Representation and Violation of Preference and Perceptual Invariance in Consumer Choice,” Organizational Behavior and Human Decision Processes, 67 (3), 280-93.

Dhar, Ravi, Stephen M. Nowlis, and Steven J. Sherman (1999), “Comparison Effects on Preference Construction,” Journal of Consumer Research, 26 (3), 293-306.

Houston, David A. and Steven J. Sherman (1995), “Cancellation and Focus: The Role of Shared and Unique Features in the Choice Process,” Journal of Experimental Social Psychology, 31 (4), $357-78$.

Houston, David A, Steven J Sherman, and Sara M Baker (1989), “The influence of unique features and direction of comparison of preferences,” Journal of Experimental Social Psychology, 25 (2), 121-41. 
Jones, Edward E and Victor A Harris (1967), “The attribution of attitudes,” Journal of Experimental Social Psychology, 3 (1), 1-24.

Judd, Charles M., Carey S. Ryan, and Bernadette Park (1991), “Accuracy in the judgment of ingroup and out-group variability.," Journal of Personality and Social Psychology, 61 (3), 366-79.

Lefkoff-Hagius, Roxanne and Charlotte H. Mason (1993), “Characteristic, Beneficial, and Image Attributes in Consumer Judgments of Similarity and Preference,”Journal of Consumer Research, 20 (1), 100-110.

Linden, Greg, Brent Smith, and Jeremy York (2003), “Amazon.com recommendations: item-toitem collaborative filtering,” IEEE Internet Computing, 7 (1), 76-80.

Linville, Patricia W., Gregory W. Fischer, and Peter Salovey (1989), "Perceived distributions of the characteristics of in-group and out-group members: Empirical evidence and a computer simulation,” Journal of Personality and Social Psychology, 57 (2), 165-88.

Medin, Douglas L., Robert L. Goldstone, and Dedre Gentner (1993), “Respects for similarity,” Psychological Review, 100 (2), 254-78.

Miller, Dale T. and Leif D. Nelson (2002), “Seeing approach motivation in the avoidance behavior of others: Implications for an understanding of pluralistic ignorance,” Journal of Personality and Social Psychology, 83 (5), 1066-75. 
Park, Bernadette and Reid Hastie (1987), "Perception of variability in category development: Instance- versus abstraction-based stereotypes,” Journal of Personality and Social Psychology, 53(4), 621-635.

Park, Bernadette and Charles M. Judd (1990), "Measures and models of perceived group variability,” Journal of Personality and Social Psychology, 59 (2), 173-91.

Quattrone, George A. and Edward E. Jones (1980), “The perception of variability within ingroups and out-groups: Implications for the law of small numbers,” Journal of Personality and Social Psychology, 38 (1), 141-52.

Rubin, Mark and Constantina Badea (2012), “They're All the Same!. . but for Several Different Reasons: A Review of the Multicausal Nature of Perceived Group Variability,” Current Directions in Psychological Science, 21 (6), 367-72.

Spiller, Stephen A., Gavan J. Fitzsimons, John G. Lynch, and Gary H. McClelland (2013), "Spotlights, Floodlights, and the Magic Number Zero: Simple Effects Tests in Moderated Regression,” Journal of Marketing Research, 50(2), 277-288.

Tversky, Amos (1977), “Features of similarity,” Psychological Review, 84 (4), 327-52. 
Tversky, Amos and Itamar Gati (1982), "Similarity, separability, and the triangle inequality," Psychological Review, 89 (2), 123-54.

West, Patricia M. (1996), “Predicting Preferences: An Examination of Agent Learning,” Journal of Consumer Research, 23 (1), 68-80. 
Figure 1: Conceptual model for predicting a target's preferences for unchosen Widget B

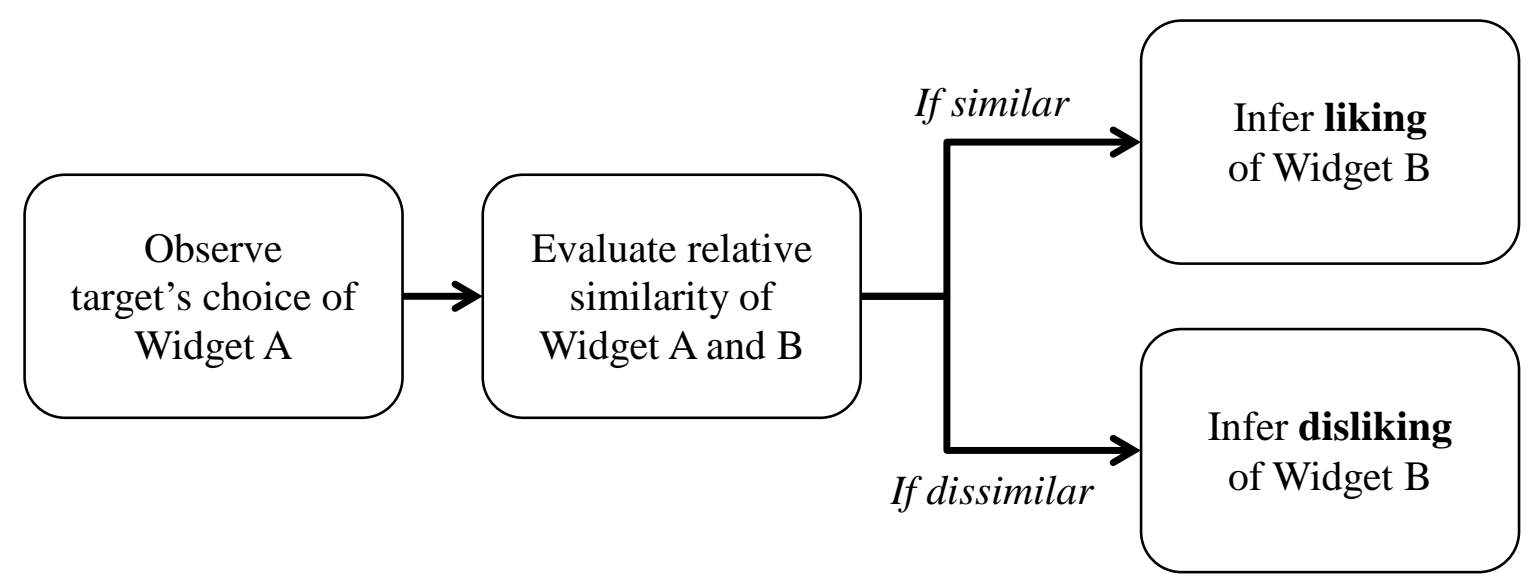


Figure 2: Study 1 Percent of participants predicting each preference for Mountain and City destinations (Clustered bars sum to 100\%; error bars depict standard errors)

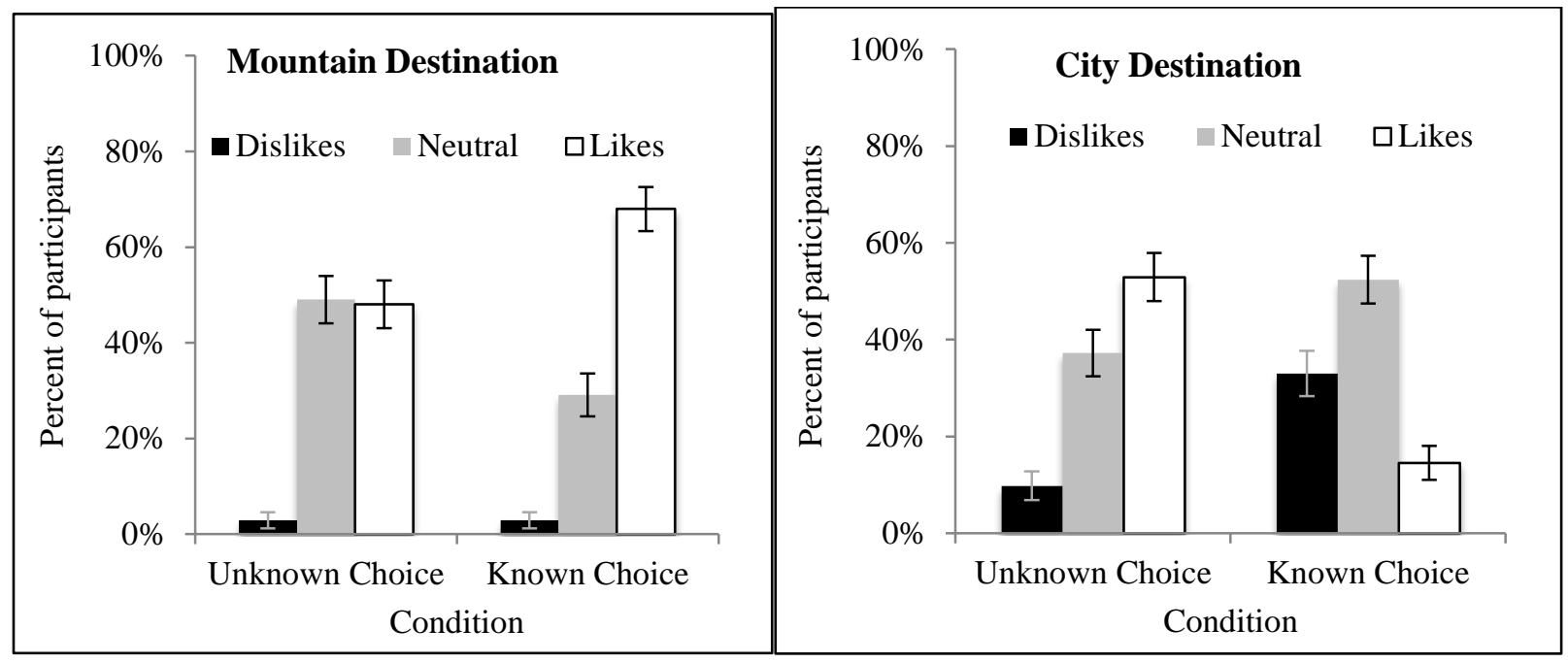


Figure 3: Study 2 Percent of participants predicting each preference for Widget B (Clustered bars sum to $100 \%$; error bars depict standard errors)

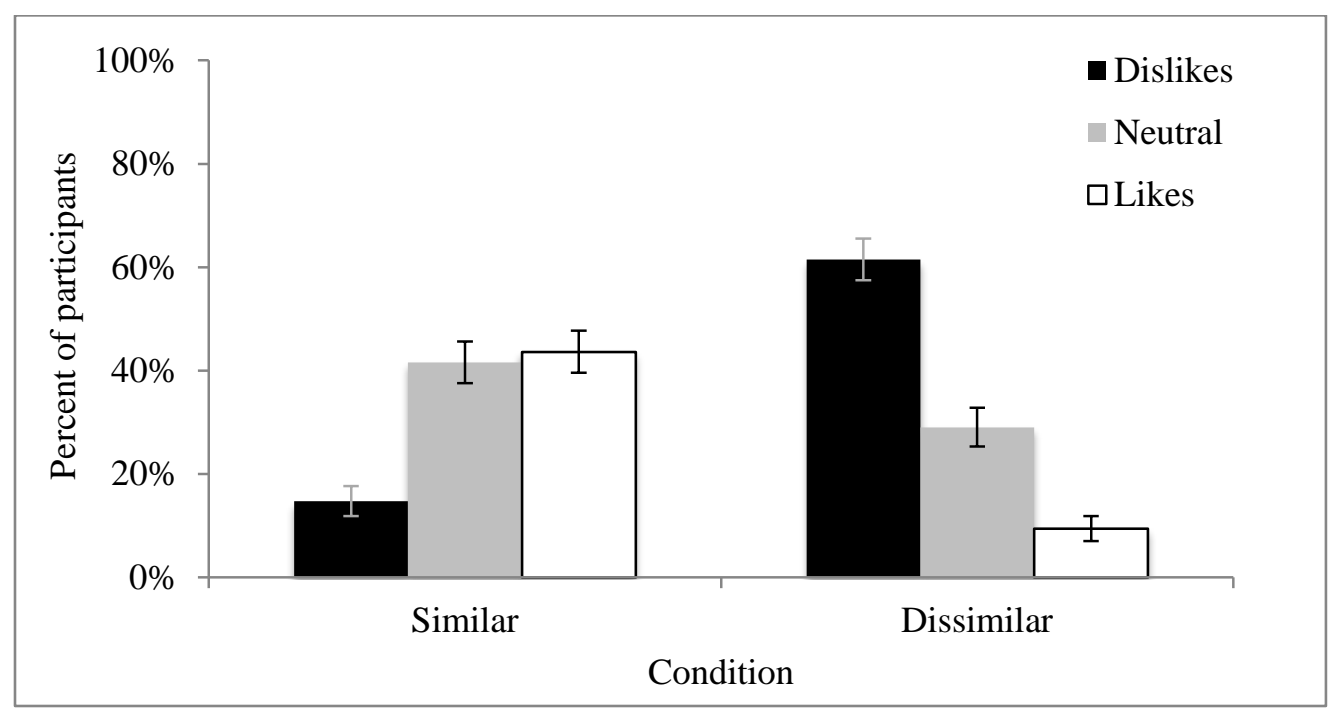


Figure 4: Study 3 Self versus observer ratings for movie genres (Error bars depict standard errors)

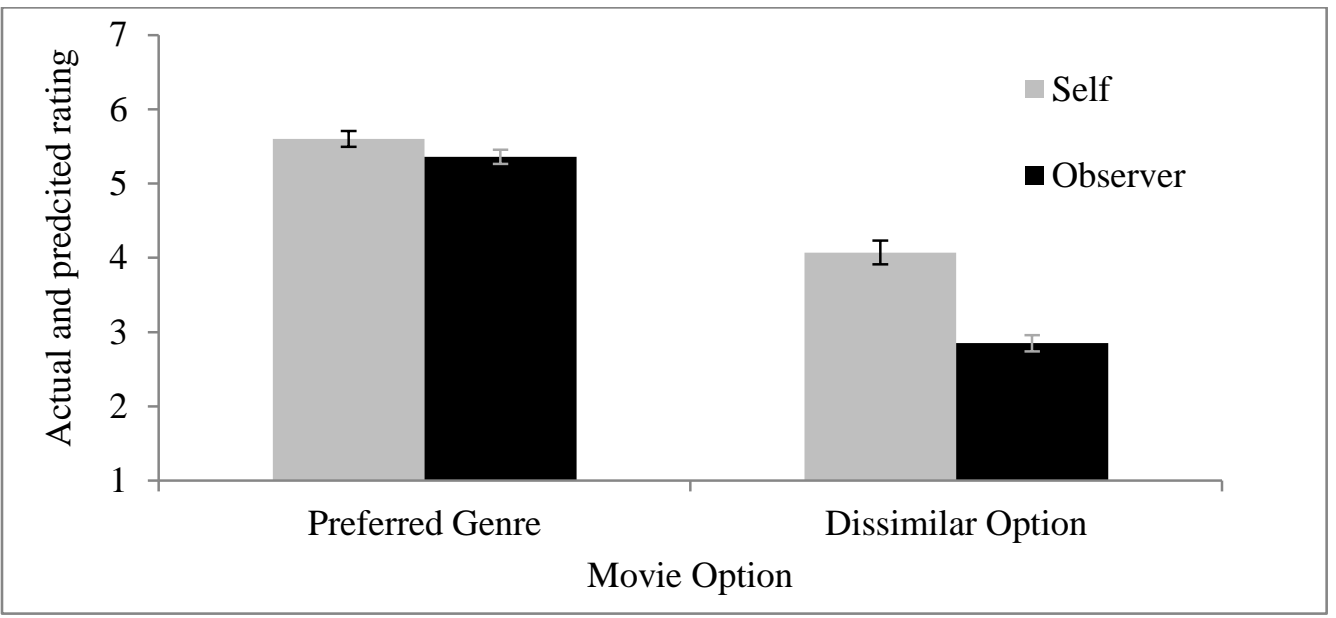


Figure 5: Study 4 Percent of participants choosing the higher rated alternative genre (Error bars depict standard errors)

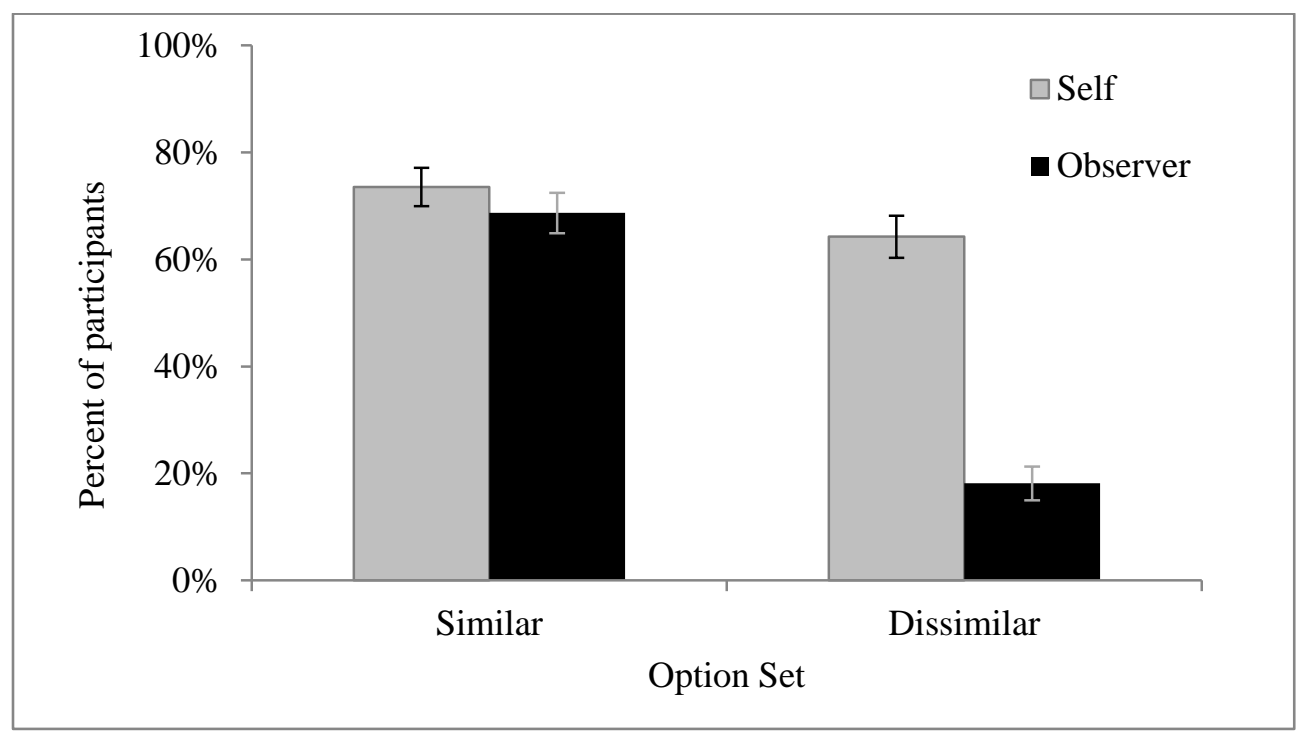


Figure 6: Study 5 Regression lines by destination option

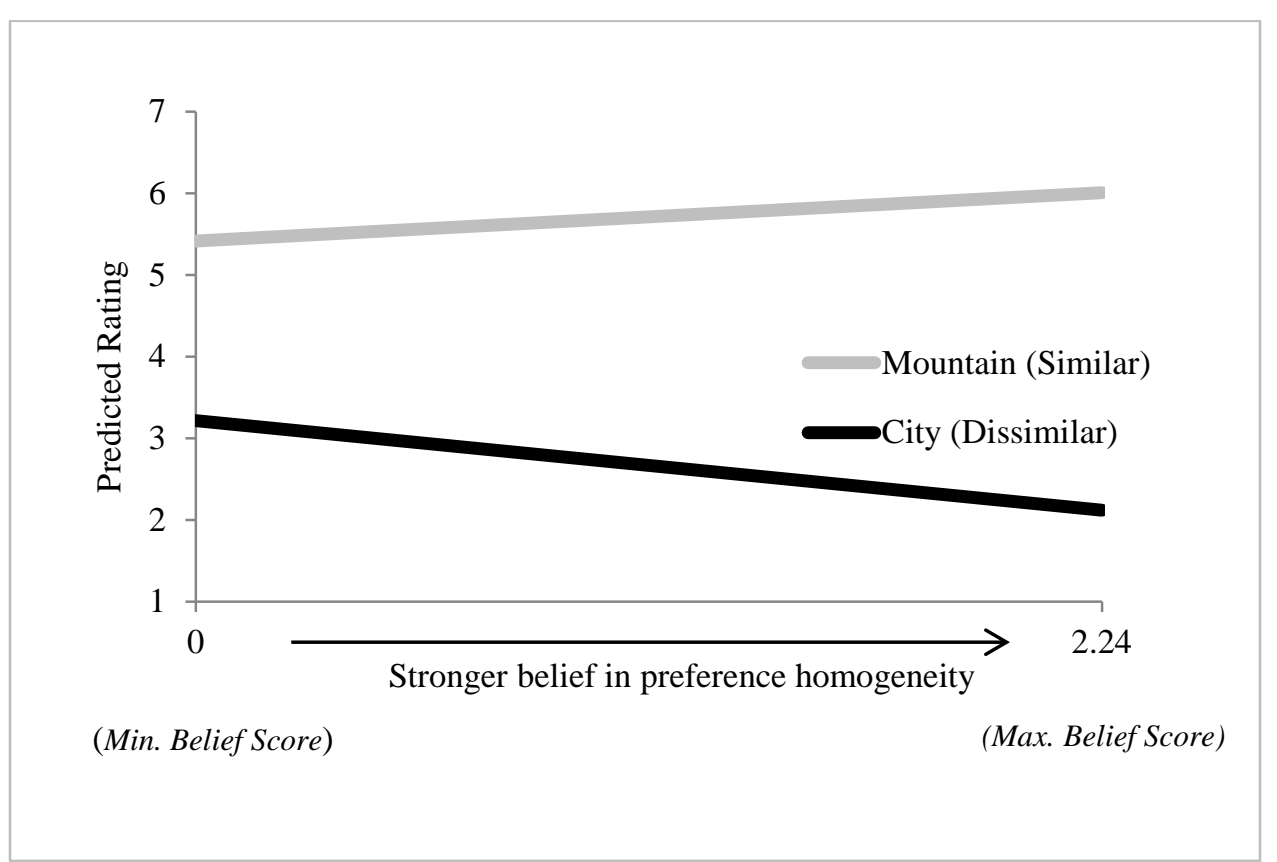

